\title{
Extending WLAN Coverage Using Infrastructureless Access Points
}

\author{
Jingyi $\mathrm{He}^{\dagger} \quad$ Jiancong Chen ${ }^{\ddagger} \quad$ S.-H. Gary Chan* \\ ${ }^{\dagger}$ Hong Kong Applied Science and Technology Research Institute Co. Ltd. \\ Shatin, New Territories, Hong Kong \\ ${ }^{\ddagger}$ Department Of Electrical Engineering, Princeton University \\ Princeton, NJ 08544, USA \\ *Department of Computer Science, The Hong Kong University of Science and Technology \\ Clear Water Bay, Kowloon, Hong Kong \\ Email: jyhe@astri.org kentchen@princeton.edu gchan@cs.ust.hk \\ Tel: +852 $23586990 \quad$ Fax: +852 23581447
}

\begin{abstract}
In wireless LAN (WLAN), the access point (AP) is connected to the Internet to provide network service to mobile users. Such system has limited coverage. To overcome it, a number of APs may form a wireless mesh and packets are forwarded from one AP to another by means of ad hoc connections. In this way, WLAN coverage can be costeffectively extended to infrastructureless areas of low population and accessibility so long as one AP (namely, I-AP) is attached to the wired infrastructure.

Since each AP can use multiple channels, we investigate in this paper the issue of routing and channel assignment in such an AP mesh network. We propose a routing scheme which takes the traffic load into consideration and routes the traffic through a load balanced shortest path tree (LB-SPT) rooted at the I-AP. We also propose a load-based channel assignment (LCA) scheme to judiciously assign the channels among the links. Our schemes are shown to significantly improve the system throughput and flow fairness, as compared with generic shortest path routing and uncoordinated random channel assignment.
\end{abstract}

Index Terms-Wireless LAN, ad hoc network, IEEE 802.11, routing, channel assignment, coverage extension.

\section{INTRODUCTION}

In recent years, we have witnessed the wide-spread deployment of IEEE 802.11-based wireless LAN (WLAN) because of its providing high-speed Internet access to mobile users. In a WLAN, an access point (AP) relays the traffic between the mobile users and the wired network it attaches. This form of setup limits the mobile users at most one hop away from the wired network. In order to extend WLAN coverage to some farther areas of lower popularity and accessibility (such as tennis courts, backyards, and remote villages), cables need to be laid to set up the wired infrastructure, which may be inconvenient or costly. In this paper, we propose a network architecture which does not require every AP to be connected to the wired network, thus able to extend the WLAN coverage cost-effectively.

In the proposed wireless mesh network, the APs are connected with each other via radio links operating on different channels from those used by the WLANs, thus forming an overlayed wireless mesh network above the WLANs. As long as an AP is connected to the wired infrastructure, the so-called infrastructure AP or I-AP, the network can offer Internet access by means of multiple hops to all the other WLANs. An example of such a network is shown in Figure 1, where WLAN is used as the access network for homes and the proposed wireless mesh network works as the "backbone" providing Internet connection to the WLAN APs. Clearly, the overlay

This work has been supported, in part, by the Hong Kong Areas of Excellence (AoE) Scheme on Information Technology of the University Grant Council (AoE/E-01/99), by HKUST postdoctoral fellowship, and by a grant of High Impact Area at HKUST (HIA03/04.EG04).

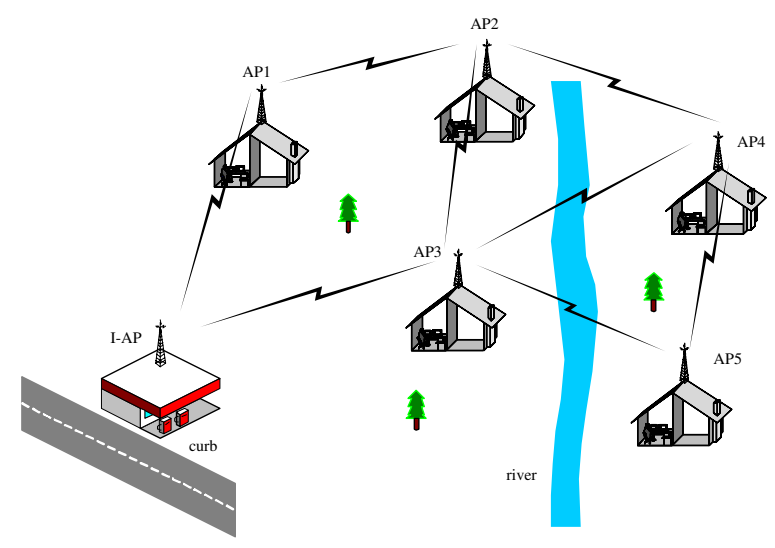

Fig. 1. A wireless mesh network composed of WLAN APs connected by wireless links.

wireless mesh is like a traditional ad hoc network if all the radio links (or APs) operate on the same channel. To attain a higher capacity, the APs may be equipped with multiple wireless interfaces so that different links may operate on different channels and transmit packets simultaneously.

In this paper, we study the issue of routing and channel assignment in such a wireless mesh network. Specifically, we consider the problem that, given a certain number of channels in the AP network, what the routes should be and how the channels should be assigned to each wireless link. The performance metrics of interest are throughput, and fairness among flows.

We propose a routing scheme which takes the traffic load of each AP into consideration. In particular, the routing is based on a load balanced shortest path tree (LB-SPT) rooted at the I-AP. We show that routing through the tree achieves high system throughput, as well as good fairness among the connections. We also propose a channel assignment scheme which gives priority to those heavy-loaded links so that they suffer less interference. This significantly improves the system throughput and fairness among the connections, as compared with an uncoordinated random assignment.

We briefly discuss related work as follows. Extending coverage in WLAN through multihop connection has been proposed in [1][3], where a mobile station outside the coverage of an AP may connect to the AP using multiple hops through other stations inside the cell. However, such an approach depends on the existence of the relay stations, and hence the coverage extension is not always reliable. Moreover, their work mainly focuses on the cooperation 
of the infrastructure mode and ad hoc mode at the relay agent. Our work, on the other hand, focuses on the routing and channel assignment issues. Our idea is similar to the multi-layer ad hoc network architecture where the upper layer acts as the backbone for transporting the traffic of the lower layers [4]. Their routing is more complex due to mobility consideration. On the other hand, since our APs are stationary, we present a much simpler routing which takes traffic load into account. A proposition for interconnecting WLAN APs that has attracted much attention recently is the mesh mode operation of the IEEE 802.16 broadband wireless access (BWA) network (also known as "WiMax" in the industry) [5]. However, such a network operates based on a different (scheduling based) MAC than IEEE 802.11. In contrast, our proposition is based on the ad hoc mode of IEEE 802.11, thus provides better upgradability for legacy APs. Actually, observing the need for "freeing the APs" from wires, the ESS Mesh Networking Task Group (TGs) has been formed recently in the IEEE 802.11 standard committee. However, the work is still in the call for proposal stage [6]. Our work presents viable routing and channel assignment solutions for such mesh networking, and studies their performances. Microsoft proposes a protocol for multichannel wireless mesh network [7]. While their work focuses on the channel selection on wireless links given the topology and channel assignment, our work studies how to come up with an appropriate network connectivity (i.e., topology) for routing and assign channels correspondingly.

The rest of this paper is organized as follows. We first discuss the routing and channel assignment problems in the proposed system in Section II. In Section III, we present our proposed routing and channel assignment algorithms. We finally present some illustrative simulation results on the system performance in Section IV, followed by conclusions in Section V.

\section{The Routing And Channel Assignment Problems}

The use of multiple channels in the proposed wireless mesh network makes routing coupled with the channel assignment at the APs. In particular, the network connectivity (and hence routing) is dependent on the channel assignment. On one hand, two APs within the transmission range of each other may not have a direct wireless connection if their wireless interfaces are assigned different operating channels. On the other hand, they may also be able to hear each other on multiple channels, or in other words, there may be multiple direct connections between them.

To make the problems more tractable, we first determine the routing assuming that there exists a direct link between two APs as long as they are within the transmission range of each other. Then we determine how to assign the channels so that the desired routing can indeed be supported while the channels are used efficiently.

\section{A. The Routing Problem}

Let $G=(V, E)$ denote the reachability graph of the wireless mesh network, where $V$ is the set of APs, and $E$ is the set of candidate wireless links. By a candidate wireless link here we mean that the two APs of the link are within the transmission range of each other. As we have discussed, such a candidate link may either not exist or represent multiple connections. The routing problem is to find the desired routes based on the reachability graph.

Since in the wireless mesh network all the traffic is between the I-AP and other APs, with shortest path routing, all the routes should constitute a shortest path tree (SPT) rooted at the I-AP. In wireless networks, the path distance is generally in terms of hop counts. The shortest path between a source-destination pair is in general not unique. Correspondingly, there are in general multiple SPTs rooted at the I-AP. If generic SPT algorithms (e.g., Dijkstra's) are used to compute the desired SPT, which one is selected is rather arbitrary. It is possible that with the computed SPT much traffic is routed through the same link, leading to high packet loss rate. For better system performance, therefore, load balancing among the links is desirable to avoid unnecessary packet losses. Our objective is to build a loadbalanced SPT (LB-SPT), in which the branches at each node are load balanced.

The load of a link is the sum of the traffic between its downstream APs and the I-AP. In this study, we consider the traffic load being the long-term pattern, based on either statistics or estimation. Routing through the LB-SPT computed based on such information can therefore ensure good long-term system performance.

\section{B. The Channel Assignment Problem}

After having the desired routing, the channel assignment problem is to determine the operating channels of the APs such that:

- the resulting network connectivity supports the routes;

- the channels are efficiently used.

To achieve the first objective, we first assign channels to the links en route, and then determine the operating channels of the APs based on the link channel assignment correspondingly.

Achieving the second objective involves taking the channel contention into consideration. On one hand, the spatial reuse of the channels should be exploited. Specifically, when two links are far apart enough so as not to interfere with each other, they should use the same channel for simultaneous transmission so as to increase the channel efficiency. On the other hand, the difference in traffic loads among the links should be considered. This is especially important when the number of channels is limited and multiple links have to contend for the same channel in a time-sharing manner. In this case, a judicious channel assignment scheme should let a heavier-loaded link suffer less contention from others.

\section{The Proposed Routing And Channel Assignment ALGORITHMS}

In this section, we present our proposed algorithms for the routing and channel assignment problems.

\section{A. Load Balancing Rerouting}

In this paper, we consider an heuristic approach that builds an LBSPT from a SPT computed without considering the traffic load. For this purpose, we propose a load balancing rerouting (LBR) algorithm.

We denote $T$ the SPT rooted at the I-AP, built by any generic SPT algorithm. The total number of nodes (i.e., APs) in the network is $|V|=N+1$, and the nodes other than the root are indexed from 1 to $N$. The number of links at the root is denoted as $M$, and the subtrees (branches) are denoted as $T_{m}, m=1, \ldots, M$. The depth of $T$ is assumed to be $L$ hops. The set of nodes on $T_{m}$ at depth $l$ is denoted by $V_{m, l}, l=1, \ldots, L$. The traffic pattern between the I-AP and other APs is denoted by $P$. For each node $n$, we assign a weight $w_{n}$, which is the aggregate traffic of itself and all its downstream nodes, or the traffic load to the upstream link of node $n$.

Obviously, the first-hop links at the I-AP have the highest traffic loads. Therefore, we first try to balance the traffic among them. The idea is to find the two links of the highest and lowest load respectively, and then shed load from the highest one to the lowest one. For example, the highest load is $w_{\max }$ from subtree $T_{i}$, and the lowest load is $w_{\text {min }}$ from subtree $T_{j}$. We then check if there is any node on $T_{i}$ at depth $l+1$ that can reach some node on $T_{j}$ at depth $l$. If there exist such two nodes $n_{1} \in V_{j, l}, n_{2} \in V_{i, l+1}$ and 
$w_{n_{2}}<w_{\max }-w_{\min }$, then we can detach $n_{2}$ from $T_{i}$ and reattach it to $T_{j}$ at $n_{1}$, by which the highest link load is reduced. Then we update the tree information, find the new $w_{\max }$ and $w_{\min }$ and repeat the above process. This process is repeated until the highest load among the first-hop links cannot be further reduced. Note that in the attempt to transfer load from subtree $T_{i}$ (of load $w_{\max }$ ) to subtree $T_{j}$ (of load $\left.w_{\text {min }}\right)$, it may be unsuccessful, because there are not two nodes on the two subtrees appropriate for the reattachment. In this case, we try the subtree of the next minimum load, until a success is achieved or all the subtrees have been tried without success. After balancing the loads of the first-hop links, the same process is recursively applied to the subtrees. Eventually, we come up with a load balanced shortest path tree rooted at the I-AP. The detail of the LBR algorithm is shown in Algorithm 1. The complexity of the algorithm is $O\left(N^{3}\right)$ (analysis omitted for limited space).

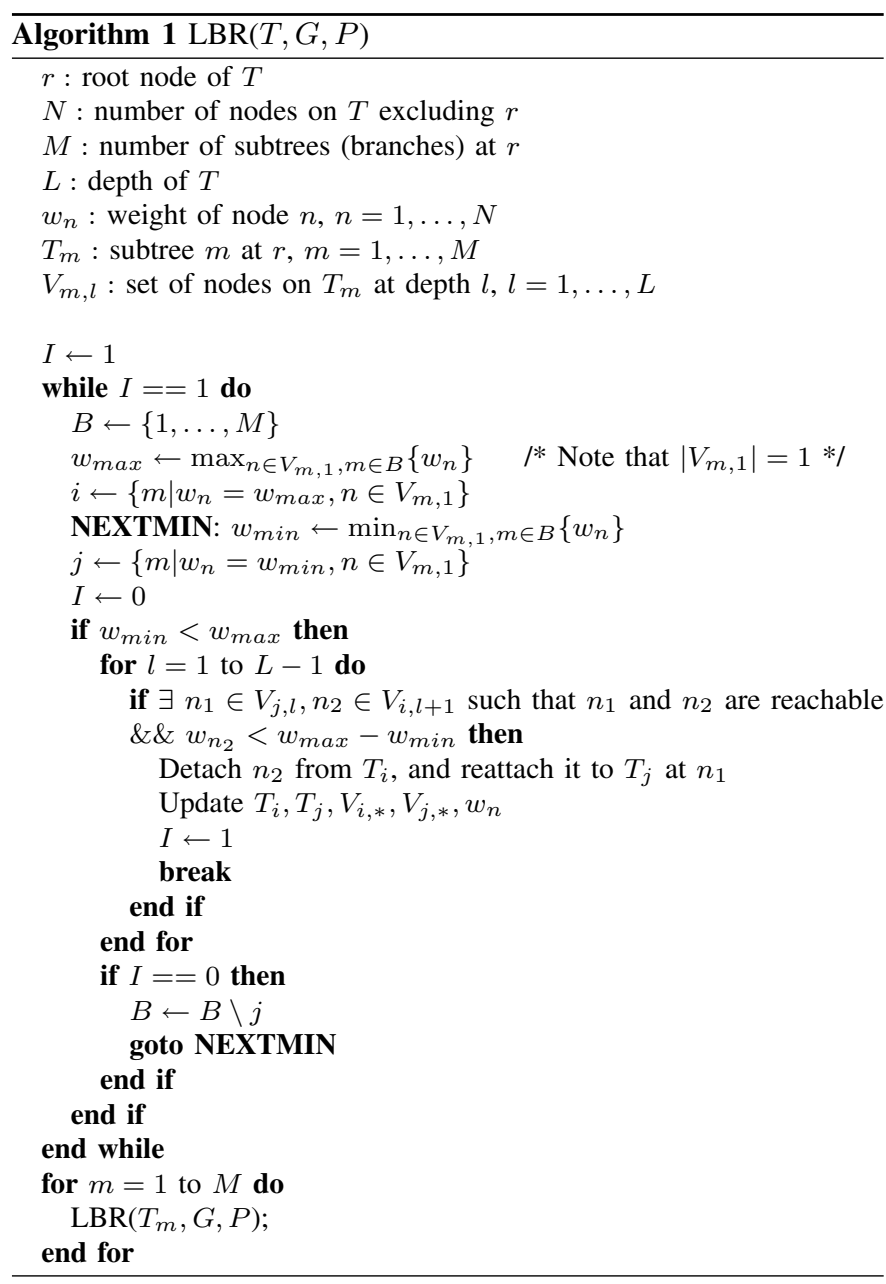

\section{B. Load-based Channel Assignment}

Given the LB-SPT and a certain number of channels, we propose the following load-based channel assignment (LCA) algorithm to assign the channels to the links on the LB-SPT.

Let $W$ denote the number of channels, and $N$ the number of links on the LB-SPT. The channel assigned to link $n$ is denoted by $a_{n}$, with $a_{n} \in[1, W]$ for $n=1, \ldots, N$. The links are sorted in decreasing order of their traffic loads, which can be calculated given the traffic matrix $L$ and the routes (i.e., the LB-SPT). If two links have the same load, the one with smaller hop count (from the I-AP) is given smaller index. Further tie is broken arbitrarily. Let $L L_{n}$ denotes the traffic load of link $n, n=1, \ldots, N$. Then we have $L L_{1} \geq \cdots \geq L L_{N}$. The basic idea of LCA is to give priority to links of higher loads and hence assign the channels to the links in the sorted order. During the assignment, the algorithm keeps track of the traffic load on each channel, denoted by $C L_{i}$ for $i=1, \ldots, W$. A link is always assigned the least-loaded channel so far.

To exploit the channel spatial reuse, in LCA, each time after a link (e.g., link $n$ ) is assigned a channel in the sorted order, we find a set of non-interfering links which are also mutually non-interfering (denoted by $I S_{n}$ ) and assign them the same channel. Basically, $I S_{n}$ and link $n$ form an independent set in the auxiliary interference graph $G_{t}^{\prime}$. The detail of the LCA algorithm is given in Algorithm 2. The complexity of the algorithm is $O\left(N^{2}+N W\right)$.

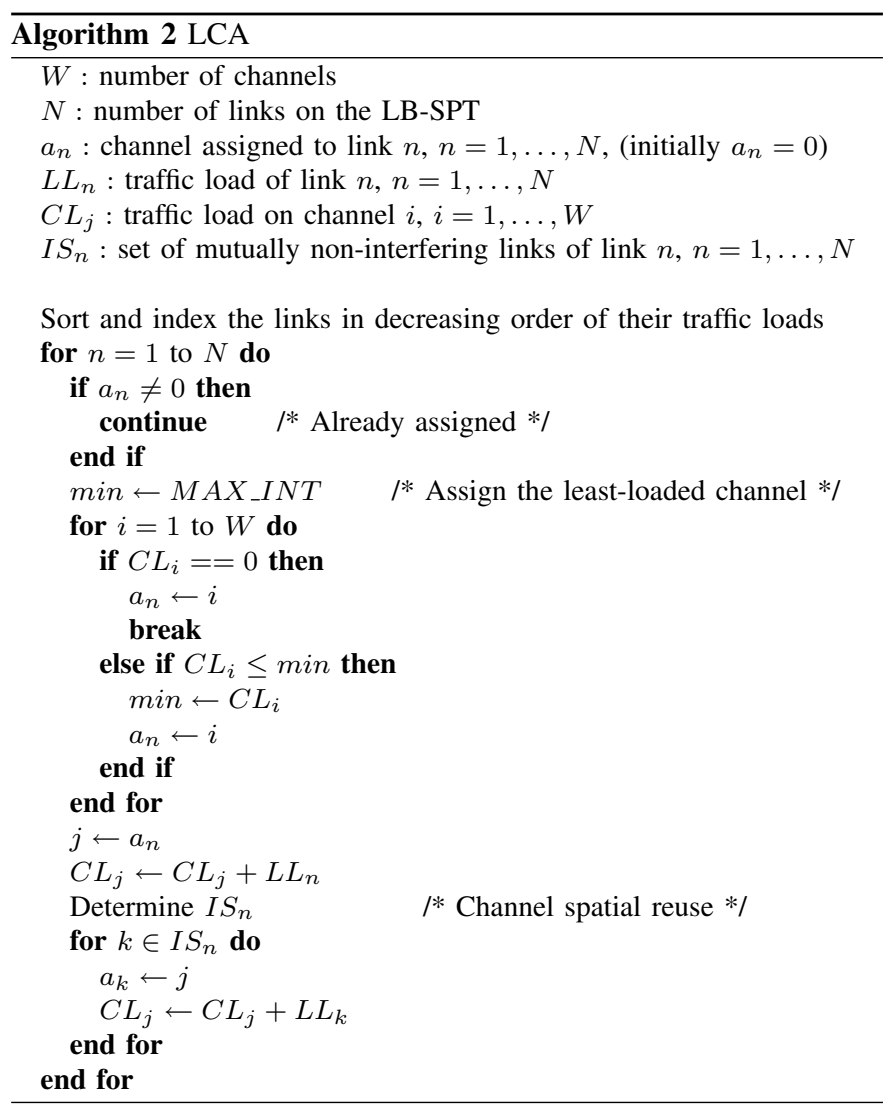

\section{ILlustrative Numerical RESUlts}

In this section, we present illustrative simulation results on the performance of the proposed multi-channel wireless mesh network, with our proposed LBR and LCA algorithms.

The performance metrics of interest include system throughput and fairness among the connections. As to the fairness, we use the Jain's fairness index [8], defined as

$$
f=\frac{\left(\sum_{i=1}^{n} x_{i}\right)^{2}}{n \sum_{i=1}^{n} x_{i}^{2}},
$$

where $n$ is the total number of flows and $x_{i}$ is the throughput of flow $i, i=1, \ldots, n$. The value of Jain's fairness index is always between zero and one, i.e., $f \in(0,1]$. A larger value means better fairness, with $f=1$ meaning perfect fairness.

We use the $n s-2$ (2.27) network simulator to do our simulations [9]. The wireless transmission rate is $2 \mathrm{Mbps}$. We consider that between each AP and the I-AP there is a duplex connection. With UDP traffic, we use constant bit rate (CBR), and all the connections have the same 


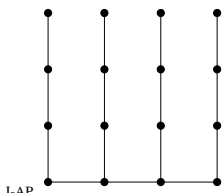

(a) $(3,12)$

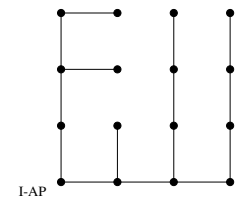

(b) $(5,10)$

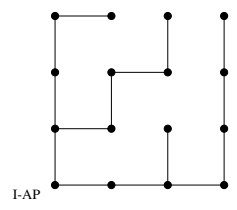

(c) $(7,8)$ by LBR
Fig. 2. The different routing selections of a $4 \times 4$ grid network, where $(i, j)$ represent the number of downstream nodes on the two branches at the I-AP.

traffic load. With TCP traffic, all the connections are bulk data transfer (e.g., FTP). In both cases, the packet size is 512 bytes. All the flows start at a random time within 1 second, and the simulation is run for 100 seconds. Note that because we are considering connecting the APs in this study, we use larger values for the transmission range and interference range of the APs than those traditionally used in WLAN and ad hoc network study (which are $250 \mathrm{~m}$ and $550 \mathrm{~m}$, respectively). In particular, we use $\sqrt{2}$ times of the traditional values, i.e., the transmission range and interference range being $354 \mathrm{~m}$ and $778 \mathrm{~m}$, respectively ${ }^{1}$.

In the following, we show illustrative simulation results of a 16node $(4 \times 4)$ grid network. The distance between two neighboring nodes is $350 \mathrm{~m}$, and the I-AP is at the left-bottom corner.

\section{A. Routing Control}

To study the impact of routing control on system performance, we consider three SPTs of different load balance levels for the 16-node grid network, as shown in Fig. 2. In the three cases, the distribution of the 15 nodes on the two subtrees at the I-AP are $(3,12),(5,10)$, and $(7,8)$, respectively. As all the nodes have the same traffic load, the $(7,8)$ distribution is the most load-balanced one. It can be derived by applying LBR to either of the other two. In this part of study, we consider 6 channels assigned to the SPTs using LCA.

We first show in Fig. 3 the system performance in the three routing cases with UDP traffic. The system throughput versus the traffic load is shown in Fig. 3(a). In all cases, as the offered load increases beyond a certain value, packet losses start to occur and the throughput cannot increase linearly any more. However, with a more balanced routing tree, the system can transport more traffic before packet losses occur. Alternatively, under the same traffic load, with a more balanced routing tree the system may achieve higher throughput. For example, at load $1400 \mathrm{Kbps},(7,8)$ by LBR achieves a throughput higher than $(3,12)$ by nearly $40 \%$. Note that as the offered load further increases, the three routing trees will eventually achieve the same system throughput, because the system capacity is determined by the two first-hop links at the I-AP with the given traffic pattern. Nevertheless, the low-loss region with moderate loads is where a real system should operate, and hence is more important. We have shown that LBR substantially improves the system throughput in this region.

The fairness index among the flows versus the traffic load is shown in Fig. 3(b). The fairness index starts to decrease when packet losses occur nonuniformly among the flows. The difference increases with the traffic load, leading to the decrease in fairness index. We see that more balanced routing tree achieves better fairness. With the most balanced $(7,8)$ routing tree by LBR, very good fairness can be achieved even at high traffic loads.

With TCP traffic, we observe that the system throughput is independent of the selection of different routing trees. This is because the TCP traffic always fully exploits the system capacity, which is

${ }^{1}$ The specific value of $\sqrt{2}$ results from the relationship between the distance of neighboring APs and the WLAN coverage range when APs are placed in grid manner to provide WLAN service fully covering a certain region [10].

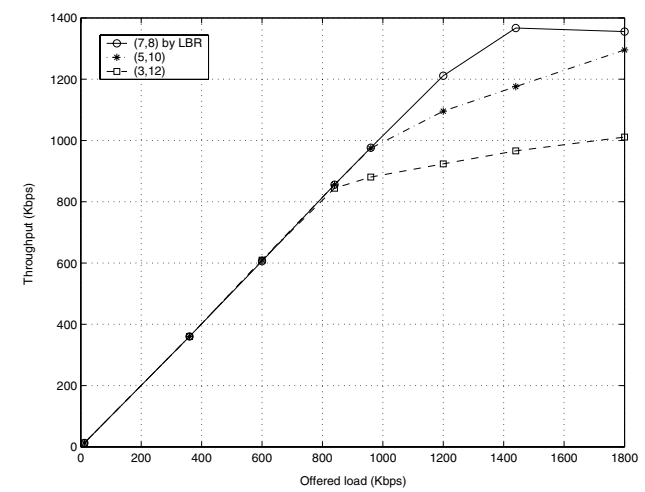

(a) System throughput

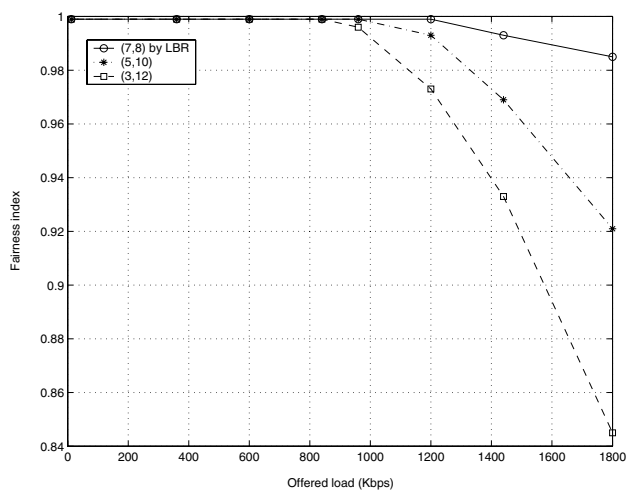

(b) Fairness index

Fig. 3. System performance under different routing, with varying UDP traffic load.

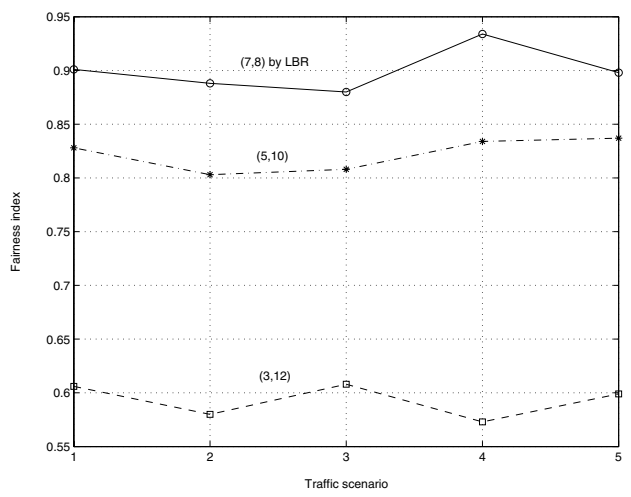

Fig. 4. Fairness index among the connections under different routing, with TCP traffic.

limited by the two first-hop links at the I-AP and hence the same for all the routing trees. Nevertheless, a load balanced routing tree is still advantageous because it can substantially improve the fairness index among the flows, as shown in Fig. 4. In this figure, the fairness index is plotted versus the traffic scenario, each of which is randomly generated with different start times of the flows.

\section{B. Channel Assignment}

In this part, we show the performance of the LB-SPT (as shown in Fig. 2(c)) when different channel assignment schemes are used. For comparison with our proposed LCA, we also consider a simple random channel assignment (RCA) scheme. Given $W$ channels and $N$ links, RCA randomly assign a channel to each link under the constraint that each channel can be assigned to at most $\lceil N / W\rceil$ links. 


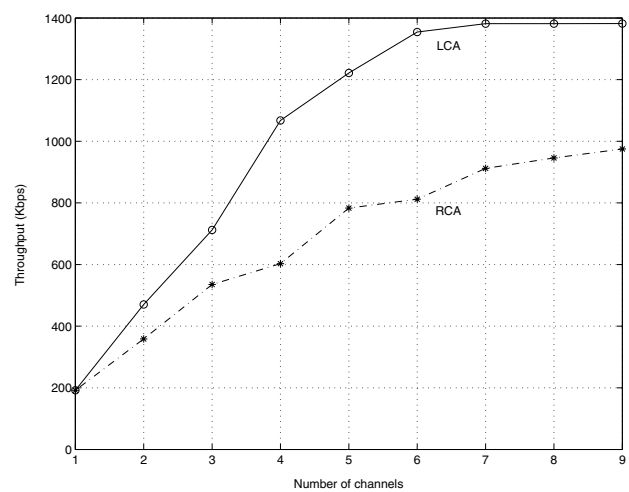

Fig. 5. System capacity as the number of channels increases and assigned by different schemes, with UDP traffic.

The constraint guarantees that every channel is used on one hand, and uniformizes the load (in terms of the number of links) on the channels on the other hand. In the following, each point in the RCA curves is an average of 10 random assignments.

We show in Fig. 5 the system capacity with UDP traffic under the two assignment schemes, as the number of channels $(W)$ varies. As can be expected, the system capacity increases with the number of channels no matter which assignment scheme is used. Nevertheless, the increase is much faster with LCA than with RCA, and hence LCA consistently achieves higher throughput than RCA when $W>1$. For example, at $W=6$ the improvement is nearly $75 \%$. Note that the throughput achieved by LCA at $W=6$ is already very close to the throughput that can be achieved with all links active simultaneously (when $W=9$ and assigned by LCA), meaning that LCA makes effective use of the channel bandwidth and hence reduces the channel requirement.

We finally show the system performance versus the number of channels with TCP traffic in Fig. 6. Similar to the case with UDP traffic, LCA makes better use of the channel bandwidth and hence achieves higher system throughput than RCA, as shown in Fig. 6(a). Different than the UDP case, with LCA the maximum system capacity is achieved with much fewer channels. As shown in the figure, the system throughput is stabilized when $W \geq 3$. This is because TCP is greedy for bandwidth, and shorter TCP connections tend to win the contention for bandwidth. Therefore, the TCP connections at the first hop of the I-AP can easily grab all the bandwidth of the channels assigned to the links therein, thus achieving the system capacity. When $W$ is small, this means farther links can rarely access the channel, leading to bad fairness. As $W$ increases, however, the fairness can be substantially improved, as shown in Fig. 6(b). With RCA, there is chance that the first-hop links at the I-AP are assigned the same channel, hence having a smaller capacity. Moreover, the randomness also may cause capacity fluctuations at larger numbers of channels.

\section{CONCLUSions}

In this paper, we have proposed a wireless mesh network architecture for cost-effective WLAN coverage extension. We have studied the routing and channel assignment issues in this network. Given that all the APs are stationary, we have proposed a fixed routing scheme which takes the traffic load into consideration and builds a load balanced shortest path tree (LB-SPT) rooted at the I-AP. A load balancing rerouting (LBR) algorithm is proposed for this purpose. We show that routing through an LB-SPT may significantly improve the system throughput and fairness among the flows. We have also proposed a load-based channel assignment (LCA) algorithm,

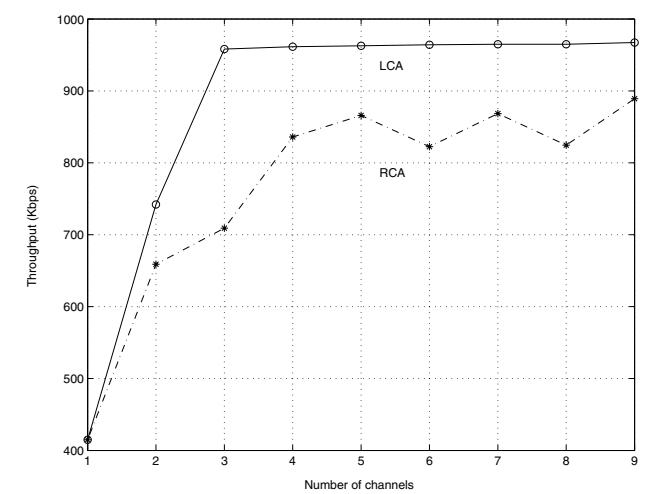

(a) System throughput

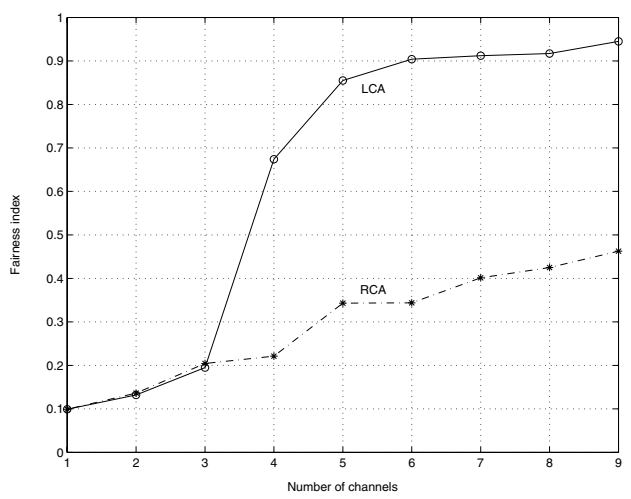

(b) Fairness index

Fig. 6. System performance as the number of channels increases and assigned by different schemes, with TCP traffic.

which significantly improves both the system throughput and fairness among the connections, as compared with an uncoordinated random assignment scheme (RCA). Future work may include adaptive routing considering dynamic traffic and load balancing when there are multiple I-APs.

\section{REFERENCES}

[1] Y.-D. Lin and Y.-C. Hsu, "Multihop cellular: A new architecture for wireless communications," in Proc. IEEE INFOCOM'O0, pp. 1273$1282,2000$.

[2] J. Chen, S.-H. Chan, J. He, and S.-C. Liew, "Mixed-mode WLAN: The integration of ad hoc mode with wireless LAN infrastructure," in Proc. IEEE Globecom'03, pp. 231-235, 2003.

[3] S. Lee, S. Banerjee, and B. Bhattacharjee, "The case for a multi-hop wireless local area network," in Proc. IEEE INFOCOM'04, 2004.

[4] K. Xu, X. Hong, and M. Gerla, "An ad hoc network with mobile backbones," in Proc. IEEE ICC'02, pp. 3138-3143, 2002.

[5] IEEE Computer Society LAN/MAN Standards Committee, "Air Interface for Fixed Broadband Wireless Access Systems - Amendment 2: Medium Access Control Modifications and Additional Physical Layer Specifications for 2-11 GHz," in IEEE Std 802.16a-2003, The Institute of Electrical and Electronics Engineers, 2003.

[6] "http://grouper.ieee.org/groups/802/11/reports/tgs_update.htm."

[7] A. Adya, P. Bahl, J. Padhye, A. Wolman, and L. Zhou, "A multiradio unification protocol of IEEE 802.11 wireless networks," Microsoft Research Technical Report MSR-TR-2003-44, Jul. 2003.

[8] R. Jain, D. Chiu, and W. Hawe, "A quantitative measure of fairness and discrimination for resource allocation in shared computer systems," in DEC Research Report TR-301, Sept. 1984.

[9] "http://www.isi.edu/nsnam/ns."

[10] A. Hills, "Large-scale wireless LAN design," IEEE Communications Magazine, vol. 39, no. 11, pp. 98-104, Nov. 2001. 